\title{
Effect of chiral interactions on the structure of Langmuir monolayers
}

\author{
E. Scalas, ${ }^{1}$ G. Brezesinski, ${ }^{2}$ V. M. Kaganer, ${ }^{2, *}$ and H. Möhwald ${ }^{2}$ \\ ${ }^{1}$ Istituto Nazionale per la Fisica della Materia, Genova, Dipartimento di Fisica Università di Genova, 16146, Genova, Italy \\ ${ }^{2}$ Max Planck Institut für Kolloid und Grenzfächenforschung, 12489 Berlin, Germany
}

(Received 5 March 1998)

\begin{abstract}
Structural changes in monolayers of the enantiomer and the racemic mixture of 1-hexadecyl-glycerol with temperature and surface pressure variations are compared. On compression, both monolayers exhibit a variation of the tilt azimuth from the direction to the nearest neighbor to the next nearest neighbor. In the monolayer of the racemate, this variation occurs as a first order transition. In the monolayer of the enantiomer, the unit cell is oblique, and continuously passes from a state close to the low-pressure state of the racemate to a state close to its high-pressure state. The azimuths of the unit-cell distortion and that of the tilt remain almost equal to each other. The effect of chirality decreases when the temperature is increased. Structural changes are explained in detail within the framework of the Landau theory of phase transitions. [S1063-651X(98)03408-4]
\end{abstract}

PACS number(s): 68.10. $-\mathrm{m}, 68.18 .+\mathrm{p}, 68.35 . \mathrm{Rh}, 64.70 . \mathrm{Md}$

\section{INTRODUCTION}

Many organic molecules are chiral: they exist in two forms called enantiomers or stereoisomers which cannot be superimposed by rotations and translations in threedimensional space. The simplest origin of chirality is the presence of a carbon atom with four nonequivalent groups attached to it. One enantiomer type predominates in the living organisms; this fact inspired many speculations on the origin of life. The 50:50 mixture of enantiomers tends to form a uniform nonchiral mixture, called a racemic mixture or racemate, because the mixing entropy is thus maximal. However, if homochiral interactions dominate, the enthalpic contribution overcomes the effect of the mixing entropy, yielding enantiomer segregation. Conversely, preferred heterochiral interactions imply the formation of a racemic compound [1].

In a monolayer system with two nonequivalent interfaces (monolayer-water and monolayer-air), only rotations about the monolayer normal, reflections in the plane containing the normal and translations in the monolayer plane, are possible symmetry operations. As a result, the monolayer system is chiral if its symmetry group does not contain a reflection in the plane containing the monolayer normal. An oblique structure in the monolayer system is chiral, and can correspond to either a monoclinic or triclinic bulk structure. Investigations of Langmuir monolayers of chiral surfactants originated from speculations that, in nearly two dimensions, it could be easier to obtain enantiomer separation due both to a lower entropic contribution induced by the reduced number of accessible conformations and to an enhancement of chiral interactions [2]. The differences in the domain shapes of racemic and enantiomeric monolayers are commonly revealed by fluorescence microscopy (see Ref. [3] for a review). However, the connection between the chirality of crystal shape and molecular shape is still a puzzle [4]. A theoretical treatment of the chiral segregation problem was given by Andel-

\footnotetext{
*Permanent address: Institute of Crystallography, Russian Academy of Sciences, 117333 Moscow, Russia.
}

man and co-workers $[1,5,6]$ who showed that, for some model interactions between headgroups, heterochiral rather than homochiral interactions are preferred.

Recent grazing-incidence $\mathrm{x}$-ray diffraction studies of Langmuir monolayers of chiral compounds showed that the relation between chirality in structure and in domain shapes is also not straightforward. Rietz et al. [7] observed left- and right-handed domains in the racemic mixture of 4hexadecyloxy-butane-1,2-diol, whereas $\mathrm{x}$-ray diffraction study reveals a centered rectangular unit cell. In monolayers of 1,2-dipalmitoyl-phosphatidylcholine, the chiral structure is observed for both enantiomer and racemate [8], even if the racemate domains do not exhibit spiral arms [9]. On the other hand, in 1-monopalmitoyl-glycerol, the structures of both the racemate and the pure enantiomer look nonchiral within experimental accuracy [10]. Heterochiral interactions prevail for most of the substances studied to date. As exceptions, Nassoy et al. [11] observed, using x-ray diffraction, the same oblique lattice in both the racemic mixture and the enantiomer of myristoyl-alanine, indicating spontaneous chiral segregation in the monolayer of the racemate. Weissbuch et al. [12] observed separation of enantiomers in the monolayers of $\alpha$-amino acid amphiphiles.

The chiral interactions are rather weak in comparison with other interactions between amphiphilic molecules, since the chiral center is only a small part of the molecule. For example, the chirality of phospholipids results in oblique unit cells quite close to the rectangular ones: the angle $\gamma$ differs from $90^{\circ}$ by only $2-3^{\circ}[8,13,14]$. Stronger effects of chiral interactions can be expected close to the phase transitions, where other interactions equilibrate each other. Such a situation is studied in the present paper: where the nonchiral monolayer of the racemate experiences a jump of the tilt azimuth at the phase transition, the monolayer of an enantiomer reveals a continuous change of the tilt azimuth, thus showing a structure which strongly deviates from that of the racemate.

The substance studied in the present paper, 1-hexadecylglycerol $\left(\mathrm{C}_{16} \mathrm{H}_{33}-\mathrm{O}-\mathrm{CH}_{2}-\mathrm{C}^{*} \mathrm{HOH}-\mathrm{CH}_{2} \mathrm{OH}\right)$ can be considered as a parent compound of phospholipids. This molecule has a glycerol headgroup with a chiral center (marked by a 
star), which is connected through an ether bond to a 16carbon chain. Preliminary investigations showed the absence of chiral segregation, despite the spiral shape of domains observed in the racemic mixture by fluorescence microscopy $[15,16]$. In the present paper, we systematically study structural changes on the temperature-surface-pressure phase diagram for both the pure enantiomer and the racemate. The structural changes are described within the framework of the Landau theory of phase transitions in Langmuir monolayers. The initial formulation of the theory $[17,18]$, which treated two different herringbone-ordered phases in the nonchiral monolayer as two independent ways of ordering of the hexatic phase, is not sufficient to describe the continuous variations between these two phases revealed by the introduction of the chiral interaction. An advanced version of the theory, which considers subsequent ordering starting from the symmetry of the isotropic two-dimensional liquid, provides an adequate description of all structural changes.

\section{EXPERIMENT}

The Langmuir monolayers of 1-hexadecyl-glycerol, kindly synthesized by $\mathrm{C}$. Tchierske at the Institute of Organic Chemistry of Halle University, Germany, were spread from a 1-mM chloroform solution onto ultrapure water purified by a Millipore desktop unit. The surface-pressure-area isotherms were recorded with a Wilhelmy balance connected to a controller (R\&K, Germany). The microscopic structure of the monolayer was investigated as a function of temperature and surface pressure at the liquid surface diffractometer on the undulator x-ray beam line BW1 at HASYLAB, DESY, Hamburg, Germany $[19,20,8]$. The synchrotron beam was made monochromatic by a Be monochromator crystal, and was deflected toward the sample and struck the air-water interface at a grazing incidence angle which is $85 \%$ of the critical angle $\alpha_{c} \simeq 0.14^{\circ}$ for total external reflection. The radiation diffracted by the interface was detected by a linear position-sensitive detector (PSD) (OED-100-M, Braun, Garching, Germany) as a function of the vertical scattering angle. A Soller collimator was located before the PSD, providing the resolution for the horizontal scattering angle. The intensities of the scattered radiation were corrected for polarization, footprint area, and powder averaging (Lorentz factor). Model peaks taken to be Lorentzian in the in-plane direction and Gaussian in the out-of-plane direction were fitted to the corrected intensities.

\section{EXPERIMENTAL RESULTS}

The phase diagrams of both the racemic mixture and the enantiomer of 1-hexadecyl-glycerol are shown in Fig. 1(a). The lines of the liquid-expanded-condensed-phase transition were obtained from surface-pressure-area isotherms [Fig. 1(b)]. At surface pressures of $15-25 \mathrm{mN} / \mathrm{m}$, a first-order phase transition between a low-pressure phase possessing tilt toward the nearest neighbors (NN's) and a higher-pressure phase with the tilt toward next nearest neighbors (NNN's) was observed in the monolayer of the racemate using $\mathrm{x}$-ray diffraction measurements, as described in detail below. The transition pressure increases as temperature increases. The transition to an untilted $(U)$ phase was visualized for the
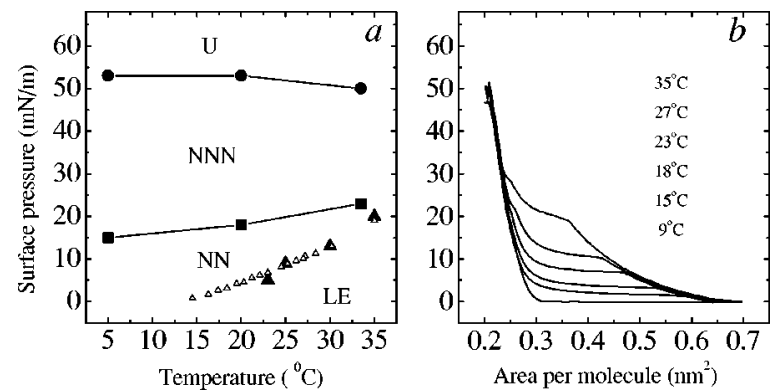

FIG. 1. (a) Phase diagram of Langmuir monolayers of racemic 1-hexadecyl-glycerol (filled symbols) with the phases: liquid expanded (LE), centered rectangular with tilt towards the nearest neighbor $(\mathrm{NN})$, towards the next-nearest neighbor $(\mathrm{NNN})$, and untilted $(\mathrm{U})$. Open triangles denote the transition from liquid expanded to condensed phase in the monolayer of the pure enantiomer; (b) the surface pressure-area isotherms of Langmuir monolayers of the enantiomer.

racemate using Brewster angle microscopy (BAM) [21]. The contrast decreases on increasing surface pressure due to the reduction of the tilt angle. A sudden vanishing of the contrast indicates the transition to the phase with upright chains. A further pressure increase leads to the appearance of bright spots of collapsed material indicating that the transition pressure is close to the collapse region of the monolayer. Therefore, it was not possible to measure the transition to the upright chain orientation directly by grazing incidence $\mathrm{x}$-ray diffraction (GIXD). In an earlier work [21] we showed that $1 / \cos \theta$, where $\theta$ is the tilt angle, is a linear function of the surface pressure and its extrapolation to zero tilt allows the estimation of the transition pressure to an untilted $(U)$ state. The transition pressures determined by BAM are in good agreement with the extrapolated GIXD values.

Figure 2 (left column) presents the contour plots of the x-ray scattered intensity at $T=5{ }^{\circ} \mathrm{C}$ for the racemic mixture. At surface pressures below the transition pressure $(15 \mathrm{mN} / \mathrm{m})$ two peaks are observed, one in the water plane $\left(Q_{z}=0\right)$ and the other one out of the water plane. This means that the molecules are tilted toward NN's. At pressures above the transition both peaks are at nonzero values of $Q_{z}$, satisfying the relation $Q_{z \mathrm{~d}}=2 Q_{z \mathrm{n}}$, where the subscript n denotes the nondegenerate and the subscript $d$ the twice more intense twofold-degenerate peak. This diffraction pattern is characteristic of NNN tilted molecules. At the transition pressure, a superposition of the peaks is observed due to phase coexistence at the first-order phase transition. The monolayer of the pure enantiomer (right column of Fig. 2) does not exhibit such a phase transition. Three peaks are observed, indicating that the chains are tilted in an intermediate direction between NN's and NNN's: the unit cell is oblique. Only far from the racemate transition pressure is the effect of chirality suppressed, and two of the three peaks merge.

From peak positions, one can determine the tilt angle $\theta$ and the tilt azimuth $\beta$, as well as unit-cell distortion magnitude $\xi$ and azimuth $\omega$ [18]. Distortion is defined in terms of the ellipse passing through the six neighbors of a given molecule: its magnitude is related to the ratio of the main axes of the ellipse, and the distortion azimuth coincides with that of the long axis. The tilt and distortion azimuths are plotted in Fig. 3 versus surface pressure for both the racemate and the enantiomer at the three investigated temperatures. For the 


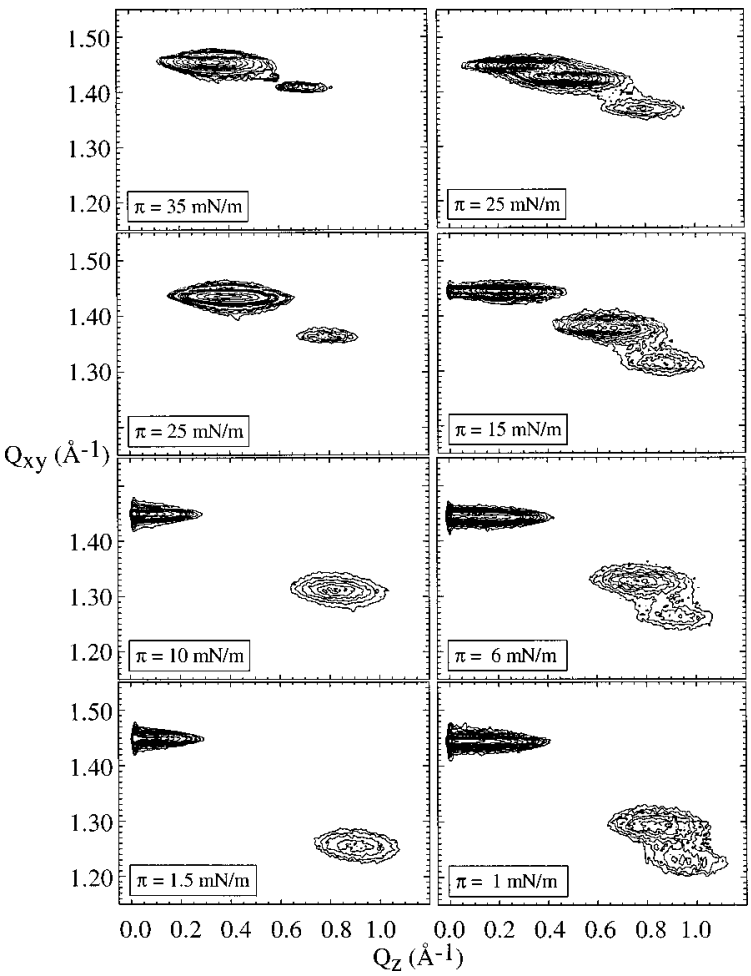

FIG. 2. Corrected x-ray intensities as functions of in-plane $\left(Q_{x y}\right)$ and out-of-plane $\left(Q_{z}\right)$ components of the scattering vector for racemic (left) and enantiomeric (right) monolayers of 1hexadecyl-glycerol at $5{ }^{\circ} \mathrm{C}$.

racemic monolayer, both azimuths point in the NN direction $\left(\beta=\omega=0^{\circ}\right)$ in the low-pressure phase and in the NNN direction $\left(\beta=\omega=30^{\circ}\right)$ in the high-pressure phase. At the transition pressures there are jumps from NN's to NNN's typical of first-order transitions. In contrast, the pure enantiomer exhibits a continuous change of both azimuths from a direction close to NN's at low pressures to one close to NNN's at pressures well above the transition pressure of the racemate. The two azimuths remain almost equal to each other $(\beta$ $\simeq \omega$ ) in the whole pressure range. One can state that the monolayer of the enantiomer experiences the same structural changes between the low- and high-pressure states as the racemate monolayer, but these changes occur continuously. From Fig. 3, one can see that the temperature increase tends to suppress the influence of chirality in the monolayer: the azimuth curves of the enantiomer monolayer become steeper as temperature increases.

By definition, the distortion $\xi$ is nonnegative, and has equal values both for a unit cell stretched and shrunk in the tilt direction. It is convenient to introduce the signed distortion $[18]$

$$
d=\xi \cos 2(\beta-\omega),
$$

which is positive when the unit cell is stretched in the tilt direction (the azimuths of the tilt and of the distortion coincide, $\beta=\omega)$ and negative when the unit cell is shrunk in this direction (the azimuths of the tilt and of the distortion are perpendicular to each other, $\beta=\omega+\pi / 2$ ). The Landau theory presented in Sec. IV treats the unit-cell distortion as a secondary effect induced by the molecular tilt and by the ordering of the hydrocarbon-chain backbones. The distortion caused by the tilt of the molecules is proportional to $\eta^{2}$

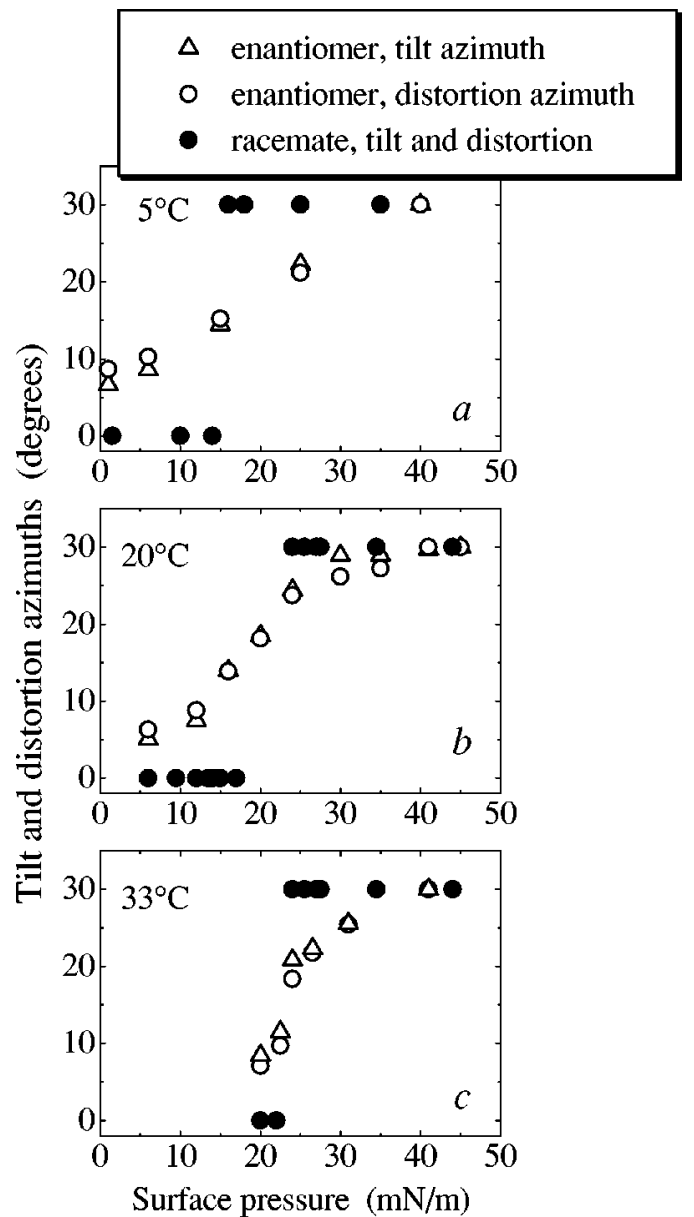

FIG. 3. Azimuths of both tilt and unit cell distortion in a chiral monolayer of 1-hexadecyl-glycerol and in the racemic mixture of its left- and right-hand enantiomers.

$=\sin ^{2} \theta$. Then, plotting the signed distortion $d$ versus $\eta^{2}$, one expects to have a straight line. The unit-cell distortion is plotted on Fig. 4 as a function of $\eta^{2}$ for both the racemate and the pure enantiomer for all the temperatures investigated. At each temperature, the values of $d$ for monolayers of both

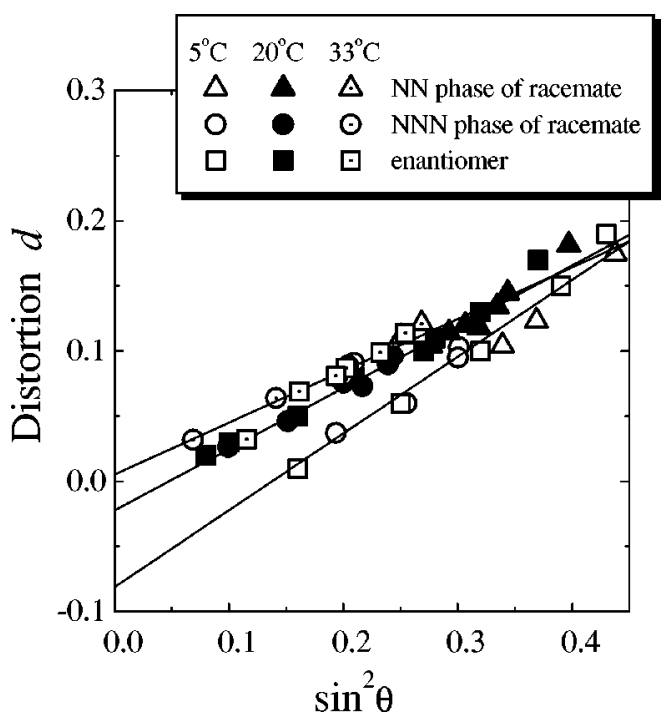

FIG. 4. The unit cell distortions versus $\sin ^{2} \theta$ at different temperatures for racemic and enantiomeric monolayers. 


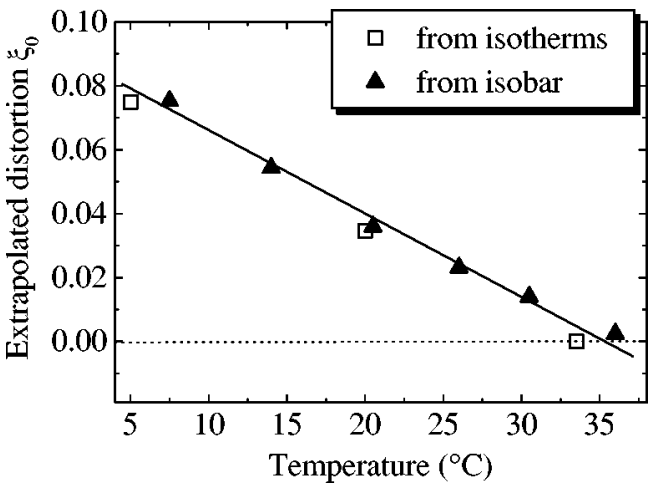

FIG. 5. The distortions extrapolated to zero tilt from the isotherms (open symbols) and the isobar at $\Pi=30.5 \mathrm{mN} / \mathrm{m}$ (filled symbols) of the racemic monolayer.

the racemate and the enantiomer lie on the same straight line. Note that the bulk crystalline structures of a racemate and an enantiomer of one and the same substance can be quite different. In the monolayer mesophases, chirality is a weak, albeit symmetry breaking, effect.

The unit-cell distortion extrapolated to zero tilt, $\xi_{0}=\left|d_{0}\right|$, is related to the degree of herringbone order of the molecular backbones, and decreases with increasing temperature. In order to follow changes in the backbone order with temperature, the structure of the racemate monolayer was determined along an isobar at $\Pi=30.5 \mathrm{mN} / \mathrm{m}$. The corresponding values of the distortion were used to determine the tiltindependent contribution to the distortion $d_{0}=d-a(T) \eta^{2}$. The slope $a(T)$ required for this determination was interpolated from the values determined along the isotherms at 5, 20 , and $33{ }^{\circ} \mathrm{C}$. The extrapolated distortion $\xi_{0}$ (Fig. 5) linearly decreases as the temperature is increased. As discussed in Sec. IV, this behavior of $\xi_{0}$ supports the treatment of distortion as a secondary effect caused by the backbone ordering. The distortion $\xi_{0}$ reaches zero at the highest temperatures which could be measured, probably indicating that the backbones become orientationally disordered at that temperature ("free-rotator"' state). One can expect that $\xi_{0}$ will remain zero upon further increase of the temperature, which was not possible to measure experimentally.

The x-ray diffraction studies of Langmuir monolayers of different nonchiral substances commonly show differences in the widths of the diffraction peaks, with the narrowest peak (and hence the longest positional correlations) corresponding to the diffraction vector normal to the tilt direction. This behavior was treated $[17,18]$ as a translational order in one direction with a herringbone-type order parameter. It is interesting to check if the direction of long correlations remains normal to the tilt direction in the oblique structures of the chiral monolayers. Having three diffraction peaks of different widths measured for the chiral monolayer of the enantiomer, we can estimate the range of positional correlations in an arbitrary direction. The correlation lengths $L$ are presented in Fig. 6 by plotting segments of length $L$ in the direction of the diffraction vectors $\mathbf{Q}$. Then ellipses passing through six points (due to three peaks of the oblique structure, with $\mathbf{Q}$ and $-\mathbf{Q}$ vectors plotted separately) are drawn. The correlation lengths are defined by the Scherrer formula $L=0.9 \times 2 \pi /$ FWHM, where FWHM is the full width at half maximum of the corresponding diffraction peak deconvolved
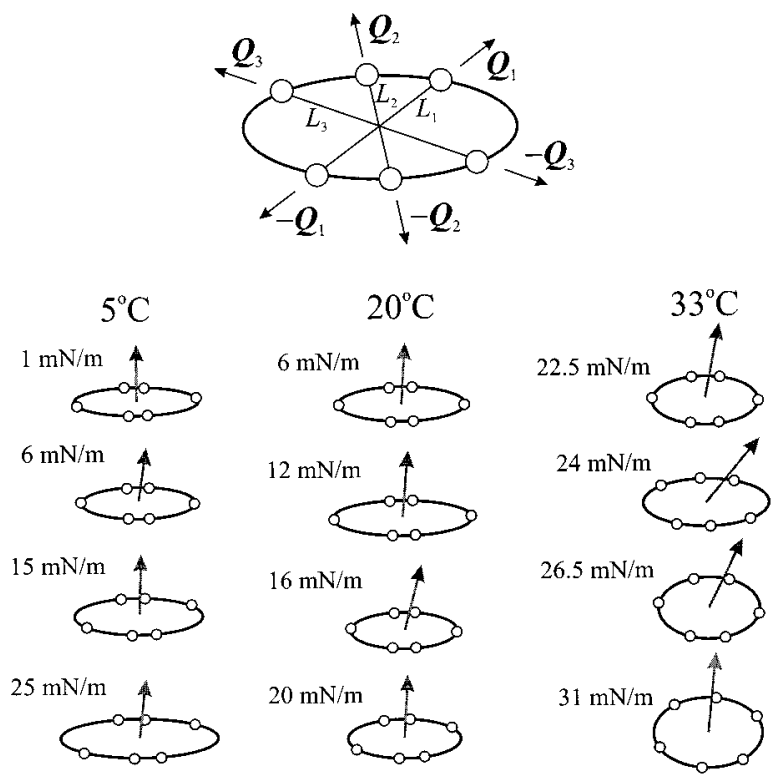

$16 \mathrm{mN} / \mathrm{m}$
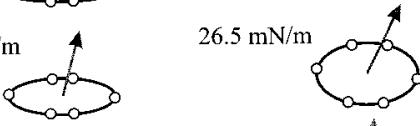

$20 \mathrm{mN} / \mathrm{m}$

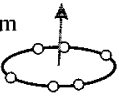

$40 \mathrm{mN} / \mathrm{m}$

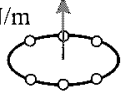

$1000 \AA$

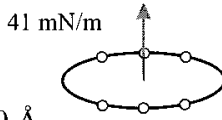

A
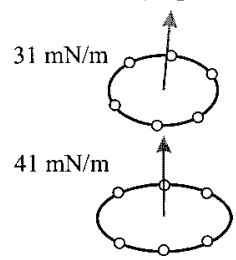

FIG. 6. Correlation lengths in the enantiomeric monolayer determined from the full widths at half maximum of three diffraction peaks of the oblique lattice. The arrows show the tilt azimuth.

with the instrumental resolution. Higher-order diffraction peaks are not observed, and we are not able to check consistency and accuracy of this construction. At temperatures of 5 and $20^{\circ} \mathrm{C}$, the aspect ratios of the ellipses vary from 2 to 4 , and their long axes remain approximately perpendicular to the tilt direction (shown by the arrows in Fig. 6). At $33^{\circ} \mathrm{C}$, stronger deviations of the tilt azimuth are observed, and the aspect ratios are smaller. A decrease of the anisotropy of positional correlations with the increasing temperature, which is expected due to a decrease of the herringbone order, follows from Fig. 6, but the effect is not pronounced.

\section{THEORY}

The x-ray diffraction data presented in Sec. III show that the nonchiral monolayer of the racemic mixture experiences a discontinuous change of the tilt azimuth from NN's to NNN's on increasing surface pressure, while the chiral monolayer of the enantiomer continuously passes from an oblique lattice with the tilt azimuth close to NN's to one close to NNN's. This behavior can be described by a simple phenomenological free energy expansion containing the tilt azimuth $\beta$ with respect to the hexatic azimuth $\gamma$ (i.e., to the mean orientation to the neighbors):

$$
\Phi=g \cos 6(\beta-\gamma)+g^{\prime} \sin 6(\beta-\gamma) .
$$

In the nonchiral monolayer, only the first term exists $\left(g^{\prime}\right.$ $=0$ ) due to reflection symmetry, which requires the free energy to be constant under transformation of the azimuths $\beta$ $\rightarrow-\beta, \gamma \rightarrow-\gamma$. Assuming that the coefficient $g$ changes sign at some surface pressure $\Pi_{0}(T)$, one finds the transition from the NN-tilted phase $(\beta=\gamma)$ at surface pressures $\Pi$ $<\Pi_{0}(g<0)$ to the NNN-tilted phase $(\beta=\gamma+\pi / 6)$ at sur- 
face pressures $\Pi>\Pi_{0}(g>0)$. A more detailed analysis $[22,17]$ involves the next-higher-order term $\cos 12(\beta-\gamma)$. Depending on the sign of the coefficient of this term, either the first-order transition at $g=0$ (as observed in the monolayer of the racemate; see Sec. III) or two second-order transitions with the intermediate tilt azimuth in between are realized.

In chiral monolayers, minimization of the free energy (2) with respect to azimuths gives $\cot 6(\beta-\gamma)=g / g^{\prime}$. Close to $\Pi_{0}$, the coefficient $g$ is proportional to $\Pi_{0}-\Pi$, whereas $g^{\prime}$ can be assumed constant. As the surface pressure is increased, the difference $\beta-\gamma$ between the tilt and the hexatic azimuths varies from 0 to $\pi / 6$, i.e., from the state close to the $\mathrm{NN}$ tilt to that close to the NNN tilt, in agreement with the experimental results. The chiral monolayer undergoes the same tilt-azimuth rotation as the nonchiral one, but, in this case, the rotation is continuous.

The simple phenomenology of Eq. (2) does not relate the transition it describes to other structural changes in the monolayer. To reveal such a relationship, we turn to the order parameters and free energy expansions introduced in Refs. $[17,18]$ from the symmetry point of view. The transition observed in the racemic mixture of 1-hexadecyl-glycerol with the change of the tilt azimuth from NN to NNN is a direct analog of the $L_{2 h}-L_{2}^{\prime}$ transition in fatty acids [18]. The phases $L_{2 h}$ and $L_{2}^{\prime}$ were treated [17] as two independent ways of ordering the backbones from the initial hexatic phase. However, in the presence of chiral interactions, the jump of the tilt azimuth is replaced by a continuous change from one backbone-ordered phase to the other one. Hence the initial formulation of the Landau theory for phase transitions in Langmuir monolayers is not sufficient. In the present section, the Landau theory is extended to explain the different backbone-ordered phases by a common origin and to provide a natural interpretation for a continuous transition between them. Here we only discuss those aspects of the theory which are essential to describe the effect of chiral interactions. A detailed analysis of the other consequences will be given elsewhere.

The theory of Refs. $[17,18]$ considered the tilt and backbone ordering occurring in the hexatic phase. The Landau free energy expansions were assumed to be invariant with respect to the symmetry of the hexatic phase. Let us now instead consider the isotropic two-dimensional liquid as the most symmetric phase. Its ordering involves not only the tilt and backbone ordering, as before, but also the hexatic ordering. Change of the azimuth of one of the order parameters with respect to the other can take place discontinuously (in the nonchiral monolayers) or continuously (in the chiral monolayers), a fact which explains the experimental observations.

The tilt order is described $[17,18]$ by the two-component order parameter $(\eta, \beta)$, where $\eta=\sin \theta$ is the magnitude $(\theta$ being the tilt angle), and $\beta$ is the azimuth of the tilt. The vector $\mathbf{n}=\left(n_{x}, n_{y}\right)$ with the Cartesian components $n_{x}$ $=\eta \cos \beta, n_{y}=\eta \sin \beta$ is the projection of the unit vector directed along the long axis of the molecule onto the plane of the monolayer. Analogously, the hexatic order is described by the two-component order parameter $(h, \gamma)$, where $h$ is the strength of the hexatic order and $\gamma$ is the azimuth of the hexatic axis. The hexatic order parameter enters the free en- ergy expansions only in combinations $h_{x}=h \cos 6 \gamma$ and $h_{y}$ $=h \sin 6 \gamma$, as a sixfold rotation $(\gamma \rightarrow \gamma+\pi / 3)$ does not change the hexatic order. Then the coupling between the tilt and the hexatic order in a nonchiral monolayer is given by the free energy term $\Phi_{h \eta}=-D h \eta^{6} \cos 6(\beta-\gamma)$, where $D$ is a constant. This term describes the transition between NNand NNN-tilted phases of the molecules with orientationally disordered backbones ( $L_{2 d^{-}} O v$ transition for fatty acids): when $D>0$, the minimum of the free energy is obtained for NN tilt $(\beta=\gamma)$, whereas $D<0$ gives rise to the NNN tilt azimuth $(\beta=\gamma+\pi / 6)$. The corresponding sine term is absent in a nonchiral monolayer due to reflection symmetry. Violation of reflection symmetry in chiral monolayers gives rise to the additional term $\Phi_{h \eta}^{\prime}=D^{\prime} h \eta^{6} \sin 6(\beta-\gamma)$ with the constant $D^{\prime}$ which is expected to be small in comparison with $D$ due to the weakness of chiral interactions. Thus the terms $\Phi_{h \eta}$ and $\Phi_{h \eta}^{\prime}$ provide the tilt contribution to Eq. (2).

It was argued $[17,18]$ that, in Langmuir monolayers, the ordering of the molecular backbone planes can be described as a "weak crystallization" with the appearance of the wave $\varphi \cos \mathbf{k} \cdot \mathbf{r}$ whose small amplitude $\varphi$ is the order parameter. The herringbone ordering of the backbones yields a glide symmetry in the direction of the wave vector $\mathbf{k}$. Accordingly, the order parameter $\varphi$ changes sign upon reflection in the plane containing the wave vector $\mathbf{k}$ and the normal to the monolayer plane. Then the free energy expansion does not contain a cubic term, which provides the possibility for a continuous phase transition with appearance of periodicity in only one direction in the monolayer plane. This type of order describes both splitting of the diffraction peaks (due to herringbone ordering) and highly anisotropic positional correlations in the monolayer (as a result of periodicity in one direction).

An alternative order parameter describing splitting of the diffraction peaks is distortion [23,24]. Distortion is certainly a quantity which distinguishes between the states with ordered and disordered backbones, but an attempt to use it as an order parameter in the Landau free energy expansions fails to correctly explain the tilt azimuths in the fatty-acid monolayer phases [25]. Also, it does not explain the anisotropic positional correlations in the backbone-ordered phases. It was argued [18] (see also below) that the distortion is a secondary effect at the transitions and consists of terms proportional to the squares of the primary order parameters $\varphi^{2}$ and $\eta^{2}$. This means in particular that the distortion is expected to possess a linear dependence on the temperature and surface pressure, $\xi \propto T_{c}-T, \Pi_{c}-\Pi$, as a consequence of the standard exponents in the Landau theory for the primary order parameters $\eta \propto \sqrt{\Pi_{c}-\Pi}, \varphi \propto \sqrt{T_{c}-T}$.

Denoting the azimuth of the wave vector $\mathbf{k}$ by $\delta$, the coupling of the amplitude $\varphi$ of the herringbone ordering wave with the tilt and the hexatic order parameters gives rise to the free energy terms for the nonchiral monolayer $\Phi_{\varphi \eta}$ $=J \varphi^{2} \eta^{2} \cos 2(\beta-\delta)$ and $\Phi_{\varphi h}=-H \varphi^{2} h \cos 6(\gamma-\delta)$. In chiral monolayers, the corresponding sine terms with coefficients $J^{\prime}$ and $H^{\prime}$ have to be added. Again, $J^{\prime}$ and $H^{\prime}$ are expected to be small in comparison to $J$ and $H$, as the chirality effect is weak. The experimental data presented above show that both NN- and NNN-tilted phases of the racemate, as well as the oblique phase of the enantiomer, 
possess long correlations in the direction orthogonal to that of the tilt. One can conclude therefore that the coefficient $J$ in the term $\Phi_{\varphi \eta}$ is positive and large enough that the minimum is at $\beta-\delta=\pi / 2$. Then the contribution to the free energy (2) from the backbone ordering is due to the terms $\Phi_{\varphi h}=H \varphi^{2} h \cos 6(\beta-\gamma) \quad$ and $\quad \Phi_{\varphi h}^{\prime}=H^{\prime} \varphi^{2} h \sin 6(\beta-\gamma)$. Generally speaking, contributions from the terms of the order $\varphi^{2} \eta^{4} h$ and $\varphi^{4} \eta^{2} h$ have to be considered as well. However, they do not alter the general qualitative conclusion. Along an isotherm, the herringbone order parameter $\varphi$ is constant, whereas the tilt order parameter $\eta$ increases upon decreasing the surface pressure. The backbone order contribution to the coefficient $g$ in Eq. (2) is dominant at small tilt angles and hence $g>0$ at large surface pressures, while the tilt contribution is dominant at large tilt angles, giving rise to $g<0$ at low surface pressures. Thus, change of the sign of the coefficient $g$ can be naturally explained as due to the interplay between the tilt and the backbone order.

Since the direction of the crystallization wave (or, in terms of the experiment, direction of the longer positional correlations) remains perpendicular to the tilt direction, one can treat structural transformations as change of the hexatic azimuth with respect to other azimuthal types of order. This change is continuous in the chiral monolayer and discontinuous in the nonchiral one. Such a treatment also explains variations of the phase diagrams in the fatty-acidalcohol mixtures [26]. The change of hexatic azimuth takes place along two segments of the fatty-acid phase diagram, $L_{2}^{\prime}-L_{2}$ and $L_{2}-O v$, and it is not surprising that, in the mixture, these segments merge and form a single transition line separating NN- and NNN-tilted regions.

The range of surface pressures where chiral interactions have an essential effect decreases with increasing temperature [cf. Figs. 3(a)-3(c)]. This fact can be explained by the increase in rotational disorder of the backbones: when neighboring molecules spin freely and independently about their long axes, they become effectively achiral, and the influence of chiral interactions is reduced. Thus chiral interactions exist only if there is a correlation between the directions of the molecular short axes [27]. In terms of the free energy expansion (2), the backbone order contribution $H^{\prime} \varphi^{2}$ dominates in the coefficient $g^{\prime}$ and, as the herringbone order parameter $\varphi$ decreases with increasing temperature, the azimuth variation becomes steeper.

Distortion of the unit cell with respect to the hexagonal one can be described [18] by the two-component order parameter $(\xi, \omega)$ characterizing the value and the orientation of the distortion. In terms of the ellipse passing through the six neighbors of a given molecule, $\xi$ is related to the ratio of the main axes of the ellipse and $\omega$ is the azimuth of the long axis. As the azimuths $\omega$ and $\omega+\pi$ are equivalent, the distortion order parameter enters the free energy expansions only in the combinations $\xi_{x}=\xi \cos 2 \omega$ and $\xi_{y}=\xi \sin 2 \omega$. Keeping in mind that the tilt direction is always perpendicular to that of the wave vector of the herringbone order, $\beta-\delta=\pi / 2$, one has the free energy expansion [18]

$$
\Phi_{\xi}=C \xi^{2}+\left(U \varphi^{2}-V \eta^{2}\right) \xi \cos 2(\beta-\omega) .
$$

The first term is the elastic energy, $C>0$, and the second describes the coupling of distortion with the primary order parameters. Strictly speaking, for a chiral monolayer one has to add a sine term to the cosine term of Eq. (3). However, this additional contribution is small and, in contrast to Eq. (2), it has no qualitative effect. When the tilt angle is large enough and $V \eta^{2}>U \varphi^{2}$, one has the minimum condition $\beta$ $=\omega$, i.e. the azimuth of the distortion coincides with that of the tilt (the unit cell is stretched in the tilt direction). Azimuth coincidence is observed in the monolayer of 1hexadecyl-glycerol in the whole investigated range of temperatures and surface pressures (Fig. 3). One can expect a change of the distortion azimuth with respect to the tilt azimuth (i.e., shrinkage of the unit cell in the tilt direction) upon decreasing tilt angle, when the condition $V \eta^{2}=U \varphi^{2}$ is fulfilled. Such a change of the distortion azimuth is observed in fatty-acid monolayers [18,28] but is not obtained, in the available range of the surface pressures, in the monolayer of 1-hexadecyl-glycerol studied in the present paper.

Minimization of the free energy (3) as a function of $\xi$ gives the distortion $\xi=(2 C)^{-1}\left(V \eta^{2}-U \varphi^{2}\right) \cos 2(\beta-\omega)$. It is convenient to use the signed distortion $d$, Eq. (1), by multiplying $\xi$ in the last equation by $\cos 2(\beta-\omega)$. The term $\cos 2(\beta-\omega)$ is equal to either +1 or -1 , depending on the sign of $V \eta^{2}-U \varphi^{2}$, as follows from the minimization of Eq. (3) with respect to azimuths. One obtains $d=\left(V \eta^{2}\right.$ $\left.-U \varphi^{2}\right) / 2 C$. Hence the dependence of the signed distortion $d$ on $\eta^{2}=\sin ^{2} \theta$ is expected to be linear, and the intercept at $\eta=0$ is the distortion due to the backbone order, $d_{0}(T)=$ $-(U / 2 C) \varphi^{2}$. The distortion can be represented as $d$ $=d_{0}(T)+a(T) \eta^{2}$, where we have written $a(T)=V / 2 C$. The coefficient $a$ can be estimated if one assumes that the unit cell in the cross section normal to the chains does not change as the tilt angle changes. Then, for coincident tilt and distortion azimuths, the spacing in the tilt direction increases as $(\cos \theta)^{-1}$ while the spacing in the perpendicular direction is constant. The distortion in the water plane varies as $(\cos \theta)^{-1}-1$. For small tilt angles, this gives $d=d_{0}(T)$ $+\eta^{2} / 2$. The experimental data yield a linear dependence of $d$ on $\eta^{2}$ along an isotherm (Fig. 4), with slopes close to the value $\frac{1}{2}$. However, a noticeable temperature dependence of the slopes is observed, showing that the unit cell in the section normal to the chains varies (albeit slightly) along the isotherms.

The distortion $\xi_{0}$ due to backbone order is temperature dependent. Close to the temperature $T_{c}$ of the transition from the rotationally disordered phase to the herringbone-ordered phase, one can predict the temperature dependence $\varphi$ $\propto \sqrt{T_{c}-T}$, as the transition is expected to be continuous (a second-order transition is allowed by the Landau theory, and the experiments do not show any discontinuity in the tilted state of the monolayer). Then one can expect $\xi_{0} \propto\left(T_{c}-T\right)$, provided that the temperature dependence of the coefficients $C$ and $U$ is weak enough. The observed temperature dependence of the distortion extrapolated to zero tilt $\xi_{0}$ is close to linear in the whole investigated temperature range (Fig. 5), thus confirming the prediction of the Landau theory and the interpretation of distortion as a secondary effect induced by tilt and backbone ordering. The experimental data of Fig. 5 show that the investigated system possibly reaches the backbone-disordered phase $\left(\xi_{0}=0\right)$ at the highest temperatures available in the experiment. 


\section{CONCLUSIONS}

We compared structural changes in the monolayers of the enantiomer and the racemic mixture of 1-hexadecyl-glycerol on compression, and found that both monolayers experience variation from $\mathrm{NN}$ to $\mathrm{NNN}$ tilt. In the monolayer of the racemate this variation takes place as a jump from $\mathrm{NN}$ to NNN tilt at the first-order transition. In the monolayer of the enantiomer, the unit cell is oblique and continuously passes from a state close to the low-pressure state of the racemate to a state close to its high-pressure state. The azimuths of the unit cell distortion and that of the tilt remain almost equal to each other. The effect of chirality decreases when the temperature increases. This observation is qualitatively explained by an increase in rotational disorder of the backbones: when neighboring molecules spin freely and independently about their long axes, they become effectively achiral, and the influence of chiral interactions is reduced. Thus chiral interactions exist only if there is a correlation between the directions of the short axes of the molecules.

An interpolation of the unit-cell distortions measured along an isobar to zero tilt enabled us to estimate the tiltindependent contribution to distortion. It turned out to be linearly temperature-dependent. At the backbone disordering transition, this contribution vanishes. The experiments show that the system investigated possibly reaches the backbonedisordered state at the highest available temperature. From the analysis of the widths of the diffraction peaks we found that both in the racemate and in the enantiomer the direction of the long correlations is orthogonal to the tilt-azimuth direction.

To explain the continuous variation of the azimuths in the chiral monolayer, we developed a Landau theory based on the free energy expansions with respect to the isotropic twodimensional liquid as the most symmetric phase. The observed transformations can be treated as change in orientation of the hexatic azimuth with respect to the azimuth of the tilt and that of the weak-crystallization wave. The change of the azimuth is continuous in the chiral monolayer, and discontinuous in the nonchiral monolayer. Distortion of the unit cell is treated as a secondary effect caused by the primary types of order, tilt and weak crystallization, and presumes a quadratic dependence of the distortion on the primary order parameters. Such a dependence was confirmed experimentally both by the observed linear dependence of the distortion on the squared tilt angle and by the linear temperature dependence of the distortion extrapolated to zero tilt.

\section{ACKNOWLEDGMENTS}

We thank Kristian Kjaer for his help in setting up the $\mathrm{X}$-ray experiment and HASYLAB at DESY, Hamburg, Germany, for beam time and support. This work was supported by the Deutsche Forschungsgemeinschaft (G. B.), the Alexander von Humboldt Stiftung (V.M.K.), Genoa University, and the Italian Ministry for University and the Scientific and Technological Research (MURST) (E. S.).
[1] D. Andelman, J. Am. Chem. Soc. 111, 6536 (1989).

[2] E. Arnett, J. Chao, B. Kinzig, M. Stewart, O. Thompson, and R. Verbiar, J. Am. Chem. Soc. 104, 389 (1982).

[3] M. McConnell, Annu. Rev. Phys. Chem. 42, 171 (1991).

[4] J. T. Groves and H. M. McConnell, Biophys. J. 70, 1573 (1996).

[5] D. Andelman, Physica A 168, 172 (1990).

[6] D. Andelman and H. Orland, J. Am. Chem. Soc. 115, 12322 (1993).

[7] R. Rietz, W. Rettig, G. Brezesinski, W. G. Bouwman, K. Kjaer, and H. Möhwald, Thin Solid Films 284, 211 (1996).

[8] G. Brezesinski, A. Dietrich, B. Struth, C. Böhm, W. G. Bouwman, K. Kjaer, and H. Möhwald, Chem. Phys. Lipids 76, 145 (1995).

[9] V. Moy, D. Keller, and H. McConnell, J. Phys. Chem. 92, 5233 (1988).

[10] G. Brezesinski, E. Scalas, B. Struth, H. Möhwald, F. Bringezu, U. Gehlert, G. Weidemann, and D. Vollhardt, J. Phys. Chem. 99, 8758 (1995).

[11] P. Nassoy, M. Goldmann, O. Bouloussa, and F. Rondelez, Phys. Rev. Lett. 75, 457 (1995).

[12] I. Weissbuch, M. Berfeld, W. Bouwman, K. Kjaer, J. AlsNielsen, M. Lahav, and L. Leiserowitz, J. Am. Chem. Soc. 119, 933 (1997).

[13] C. Böhm, H. Möhwald, L. Leiserowitz, J. Als-Nielsen, and K. Kjaer, Biophys. J. 64, 553 (1993).
[14] F. Bringezu, G. Brezesinski, P. Nuhn, and H. Möhwald, Biophys. J. 70, 1789 (1996).

[15] R. Rietz, G. Brezesinski, and H. Möhwald, Ber. Bunsenges. Phys. Chem. 97, 1394 (1993).

[16] E. Scalas, G. Brezesinski, H. Möhwald, V. M. Kaganer, W. G. Bouwman, and K. Kjaer, Thin Solid Films 284, 56 (1996).

[17] V. M. Kaganer and E. B. Loginov, Phys. Rev. E 51, 2237 (1995).

[18] V. M. Kaganer, I. R. Peterson, R. M. Kenn, M. C. Shih, M. Durbin, and P. Dutta, J. Chem. Phys. 102, 9412 (1995).

[19] J. Als-Nielsen, D. Jacquemain, K. Kjaer, F. Leveiller, M. Lahav, and L. Leiserowitz, Phys. Rep. 246, 251 (1994).

[20] K. Kjaer, Physica B 198, 100 (1994).

[21] C. DeWolf, G. Brezesinski, G. Weidemann, H. Möhwald, K. Kjaer, and P. B. Howes, J. Phys. Chem. B 102, 3238 (1998).

[22] J. V. Selinger and D. R. Nelson, Phys. Rev. Lett. 61, 416 (1988); Phys. Rev. A 39, 3135 (1989).

[23] E. B. Sirota, H. E. King, D. M. Singer, and H. Shao, J. Chem. Phys. 98, 5809 (1993).

[24] E. B. Sirota, Langmuir 13, 3849 (1997).

[25] V. M. Kaganer and V. L. Indenbom, J. Phys. II 3, 813 (1993).

[26] B. Fisher, E. Teer, and C. M. Knobler, J. Chem. Phys. 103, 2365 (1995).

[27] A. B. Harris, R. D. Kamien, and T. C. Lubensky, Phys. Rev. Lett. 78, 1476 (1997).

[28] R. M. Kenn, C. Böhm, A. M. Bibo, I. R. Peterson, H. Möhwald, J. Als-Nielsen, and K. Kjaer, J. Phys. Chem. 95, 2092 (1991). 